\begin{tabular}{|c|c|}
\hline Title & Semi-A nalytical A pproach for Finite-Element A nalysis of Multi-Turn Coil Considering Skin and Proximity Effects \\
\hline Author(s) & Igarashi, Hajime \\
\hline Citation & $\begin{array}{l}\text { IEEE Transactions on Magnetics, 53(1), } 7400107 \\
\text { https://doi .org/10.1109/T MA G.2016.2601066 }\end{array}$ \\
\hline Issue Date & 2017-01 \\
\hline Doc URL & http:/hdl.handle.net/2115/64396 \\
\hline Rights & $\begin{array}{l}\text { (C } 2016 \text { IEEE. Personal use of this material is permitted. Permission from IEEE must be obtained for all other uses, in } \\
\text { any current or future media, including reprinting/republishing this material for advertising or promotional purposes, } \\
\text { creating new collective works, for resale or redistribution to servers or lists, or reuse of any copyrighted component of } \\
\text { this work in other works. }\end{array}$ \\
\hline Type & article (author version) \\
\hline File Information & igarashi-final.pdf \\
\hline
\end{tabular}

Instructions for use 


\title{
Semi-Analytical Approach for Finite Element Analysis of Multi-turn Coil Considering Skin and Proximity Effects
}

\author{
Hajime Igarashi, Member, IEEE
}

Graduate School of Information Science and Technology, Hokkaido University, Sapporo 060-0814, Japan

\begin{abstract}
Native application of finite element method (FEM) to analysis of skin and proximity effects in multi-turn coils results in large equation systems whose solution needs long computational time. This paper proposes a semi-analytical approach to overcome this problem. For analysis of the proximity effect, the complex permeability of a round conducting wire immersed in uniform time-harmonic magnetic fields is represented in a closed form. Then, the homogenized complex permeability over the cross section of the multi-turn coil is analytically evaluated using the Ollendorff formula. The magnetoquasistatic problem is thus replaced by the magnetostatic one in which the multi-turn coil is treated as a uniform material with the homogenized complex permeability. The skin effect is taken into consideration by introducing the corresponding impedance in the circuit equation. The proposed method is shown to give the impedance of multi-turn coils which is in good agreement with that obtained by conventional FEM as well as experiments.
\end{abstract}

Index Terms - Skin effect, proximity effect, eddy current, homogenization, complex permeability, multi-turn coil.

\section{INTRODUCTION}

$I^{1}$ $\mathrm{T}$ is of importance to numerically evaluate the eddy current losses in multi-turn coils used in electric machines and devices such as motors [1,2] and inductors [3] because of increase in the driving frequency. Moreover, development of contactless energy transfer systems [4, 5] and eddy-current sensors [6] requires to accurately compute frequency dependence of the impedance in multi-turn coils. The eddy current loss $P$ in the multi-turn coil is composed of $P_{\text {skin }}$ and $P_{\text {prox }}$ which are attributed to the skin and proximity effects, respectively. Because currents in a wire tend to localize near its surface due to the skin effect, the wire resistance increases with frequency. The proximity effect is due to the magnetic induction, $B_{0}$, generated by the currents surrounding the wire. The magnetic dipole appears to prevent the time variation of $B_{0}$ on the cross section of the wire. For this reason, the multi-turn coil has essentially diamagnetic property which has to be considered in the field analysis.

Two types of analytical approaches for evaluation of $P$ in multi-turn coils have been proposed so far. In the first approach [7], which has also been discussed in other papers as referred in [8], each column of round wires is approximately replaced by an infinitely long conducting foil. Then $P$ in each foil is analytically computed assuming that the foils are immersed in one-dimensional magnetic field. On the other hand, in the second approach [9], $P$ in an isolated round wire is evaluated neglecting the magnetic fields generated by the currents flowing along other wires. The magnetic induction $B_{0}$ which causes the proximity effect is not accurately evaluated by either of them. Indeed, it has been pointed out in [8] that the former and latter approaches under- and over-estimate $P_{\text {prox }}$.

To compute the eddy current losses using conventional FE analysis, each wire has to be subdivided into so fine elements that the element size is sufficiently smaller than the skin depth.

Manuscript received March 24. Corresponding author: H. Igarashi (e-mail: igarashi@ssi.ist.hokudai.ac.jp).

Digital Object Identifier (inserted by IEEE).
The whole cross-section of a multi-turn coil may, therefore, include huge number of finite elements. For this reason, the conventional FE analysis of multi-turn coil would need considerably long computational time.

It has been shown that $P_{\text {prox }}$ can be effectively evaluated by subdividing the cross-section of a multi-turn coil into elementary cells, each of which spans one spatial period, and performing FE analysis of quasi-static fields in the elementary cell under appropriate boundary conditions [10]. In this method, the homogenized complex permeability $\langle\dot{\mu}\rangle$ which represents the diamagnetic effect of eddy currents is evaluated from the quasi-static FE analysis. Then $B_{0}$ is determined from the magnetostatic FE analysis in which the multi-turn coil is modeled as a homogeneous material whose permeability is $<$ $\dot{\mu}>$. Moreover, on the basis of this approach, the analytical formula proposed in [9] to compute $P_{\text {prox }}$ has been extended to improve its accuracy [8]. The dependence of $P$ on the shape of the wire cross section and configuration has also been discussed [11]. Although these methods seem effective, one has to perform FE analysis to obtain $\langle\dot{\mu}\rangle$ for different frequencies and volume fraction of multi-turn coils.

In this paper, a semi-analytical approach for the eddy current analysis of multi-turn coils is proposed. In this method, from the complex permeability $\dot{\mu}$ of a round wire expressed in a closed form, the homogenized complex permeability $\langle\dot{\mu}\rangle$ over the cross section of a multi-turn coil is analytically evaluated using the Ollendorff formula [12]. Then, magnetostatic FE analysis is performed to determine magnetic fields around the homogenized coil region with permeability $<$ $\dot{\mu}>$ for computation of $P_{\text {prox }}$. On the other hand, $P_{\text {skin }}$ is evaluated by introducing the AC resistance. The FE analysis of multi-turn coils is simplified by the proposed method because it determines $\langle\dot{\mu}\rangle$ for any frequency and volume fractions without field computations. It will be shown that the impedance of the multi-turn coil computed by the proposed method agrees well with that computed by the conventional FE approach in which the each coil is subdivided into sufficiently fine FE meshes. Moreover, the coil impedance computed by the 


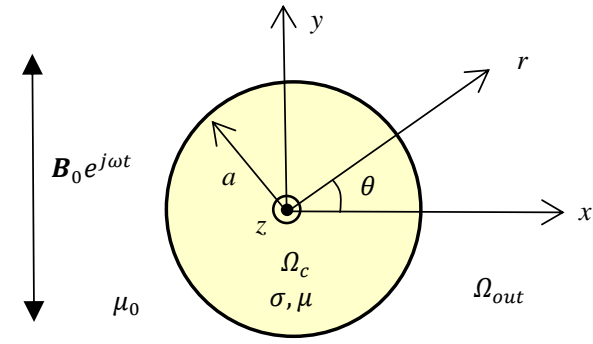

Fig. 1. Cross-section of a straight round conducting wire immersed in uniform, time-harmonic magnetic field

\section{TABLE I Nomenclature}

\begin{tabular}{|c|c|c|c|}
\hline Symbol & Quantity & $\begin{array}{c}\text { Symbo } \\
1\end{array}$ & Quantity \\
\hline$a$ & wire radius & $B$ & magnetic induction \\
\hline$\sigma$ & conductivity & $\boldsymbol{B}_{0}$ & $\begin{array}{l}\text { external uniform } \\
\text { magnetic induction }\end{array}$ \\
\hline$\mu$ & permeability & $\boldsymbol{H}$ & magnetic field \\
\hline$\mu_{0}$ & $\begin{array}{l}\text { permeability of } \\
\text { vacuum }\end{array}$ & $M$ & magnetization \\
\hline$\omega$ & angular frequency & $E$ & electric field \\
\hline$J_{n}$ & $\begin{array}{l}n \text {-th order Bessel } \\
\text { function }\end{array}$ & $A$ & vector potential \\
\hline$l$ & wire length & $m$ & magnetic moment \\
\hline$\delta$ & skin depth & $\dot{\mu}$ & $\begin{array}{l}\text { complex } \\
\text { permeability of } \\
\text { isolated wire }\end{array}$ \\
\hline$\Omega_{c}$ & wire region & $\langle\dot{\mu}\rangle$ & $\begin{array}{l}\text { homogenized } \\
\text { complex } \\
\text { permeability }\end{array}$ \\
\hline$\Omega_{\text {out }}$ & air region & $\eta$ & volume fraction \\
\hline$k$ & $\sqrt{-j \omega \sigma \mu}=(1-j) / \delta$ & $N$ & $\begin{array}{l}\text { diamagnetic } \\
\text { constant }\end{array}$ \\
\hline$z$ & $k a$ & $I$ & current \\
\hline$w$ & $\begin{array}{l}\text { scalar interpolation } \\
\text { function in FEM }\end{array}$ & $V$ & voltage \\
\hline$S_{c}$ & area of $\Omega_{c}$ & $P$ & power \\
\hline$Z_{e}$ & $\begin{array}{l}\text { impedance of } \\
\text { external circuit }\end{array}$ & $j$ & imaginary unit \\
\hline
\end{tabular}

proposed method will be shown to agree well with experimental results.

\section{FORMULATION}

The nomenclature is summarized in TABLE I.

\section{A. Electromagnetic field around a round wire}

Let us consider a round wire, carrying a uniform current, immersed in a uniform time-harmonic magnetic field $\boldsymbol{B}_{0} e^{j \omega t}$ perpendicular to the wire axis as show in Fig.1. The coordinate systems $(x, y, z)$ and $(r, \theta, z)$ are introduced where $y$ and $z$-axes are defined parallel to $\boldsymbol{B}_{0}$ and the wire axis, respectively. It is assumed that $\sigma=0$ in $\Omega_{\text {out }}$. Moreover, it is assumed that the curvature of the wire is negligible and there is no field variation in $z$ direction. We consider only $E_{z}$ as the currents flow in $z$ direction. From the Maxwell equations under the quasi-static approximation $[13,14]$

$$
\operatorname{rot} \boldsymbol{H}=\sigma \boldsymbol{E}
$$

$$
\operatorname{rot} \boldsymbol{E}=-j \omega \mu \boldsymbol{H}
$$

we obtain

$$
\frac{\partial^{2} E_{z}}{\partial r^{2}}+\frac{1}{r} \frac{\partial E_{z}}{\partial r}+\frac{1}{r^{2}} \frac{\partial^{2} E_{z}}{\partial \theta^{2}}+k^{2} E_{z}=0
$$

where $k=0$ for $r \geq a$. Applying the variable separation $E_{z}=$ $R(r) \Theta(\theta)$ to (2), we obtain

$$
\begin{array}{r}
r^{2} R^{\prime \prime}+r R^{\prime}+\left(k^{2} r^{2}-\beta^{2}\right) R=0 \\
\Theta^{\prime \prime}+\beta^{2} \Theta=0
\end{array}
$$

The current due to skin effect is free from $\theta$ whereas the eddy current, which flows in $\pm z$-directions to generate dipole magnetic fields in response to $\boldsymbol{B}_{0}$, would depend on $\cos \theta$. Hence, we consider the following solutions to (3)

$$
E_{z}=p_{0} J_{0}(\zeta)+p_{1} J_{1}(\zeta) \cos \theta, \quad r \leq a
$$

where $\zeta=k r$. The first and second terms in (4) express the skin and proximity effects. By substituting (4) into (1b), we obtain the $\theta$-component of $\boldsymbol{H}$ in the form

$$
H_{\theta}=\frac{j k}{\omega \mu}\left[p_{0} J_{1}(\zeta)-p_{1} J_{1}^{\prime}(\zeta) \cos \theta\right], \quad r \leq a
$$

When $r \geq a$, (2) reduces to the Laplace equation whose solution of our interest is written as

$$
\begin{array}{cc}
E_{z}=\left(\frac{q_{1}}{r}+j \omega r B_{0}\right) \cos \theta, & r \geq a \\
H_{\theta}=-\frac{j}{\omega \mu_{0}}\left(-\frac{q_{1}}{r^{2}}+j \omega B_{0}\right) \cos \theta, & r \geq a
\end{array}
$$

The first and second terms in (6) and (7) express the dipole field due to the eddy currents generated by the proximity effect and the uniform external field, respectively. Note here that the components independent of $\theta$, which will not appear in the formulation, are omitted in (6) and (7) for simplicity.

When the total current $I$ flowing along the wire is given, $p_{0}$ can be determined by applying the Ampere law to (5) as follows [15, sec.60]:

$$
p_{0}=\frac{k I}{2 \pi a \sigma J_{1}(z)}
$$

The constants $p_{1}, q_{1}$ can be determined from the continuity condition for $E_{Z}, H_{\theta}$ on the surface of the wire, which is expressed by

$$
\left[\begin{array}{cc}
J_{1}(z) & -1 / a \\
z J_{1}^{\prime}(z) / \mu_{r} & 1 / a
\end{array}\right]\left[\begin{array}{l}
p_{1} \\
q_{1}
\end{array}\right]=j \omega a B_{0}\left[\begin{array}{l}
1 \\
1
\end{array}\right]
$$

where $\mu_{r}=\mu / \mu_{0}$. 


\section{B. Energy conservation}

From the Maxwell equations (1), the energy conservation law

$$
\int_{\Omega_{c}}\left(\sigma|\boldsymbol{E}|^{2}+j \omega \mu|\boldsymbol{H}|^{2}\right) d v=-\int_{\partial \Omega_{c}} \boldsymbol{E} \times \boldsymbol{H}^{*} \cdot d \boldsymbol{S}
$$

can be derived. On the other hand, the power $P=V I^{*} / 2$ supplied by the external source equals the increase in the magnetic energy and eddy current loss in the wire. Hence we have $[15$, sec.60]

$$
P=\frac{j \omega}{2} \int_{\Omega_{c}+\Omega_{\text {out }}} \mu|\boldsymbol{H}|^{2} d v+\frac{1}{2} \int_{\Omega_{c}} \sigma|\boldsymbol{E}|^{2} d v
$$

Substitution of (10) into (11) yields

$$
P=\frac{j \omega}{2} \int_{\Omega_{\text {out }}} \mu|\boldsymbol{H}|^{2} d v-\frac{1}{2} \int_{\partial \Omega_{c}} \boldsymbol{E} \times \boldsymbol{H}^{*} \cdot d \boldsymbol{S}
$$

By inserting (4), (5) into (12), we find

$$
\begin{aligned}
P= & \frac{j \omega}{2} \int_{\Omega_{\text {out }}} \mu|\boldsymbol{H}|^{2} d v+\frac{R_{0} z J_{0}(z)}{4 J_{1}(z)}|I|^{2} \\
& +j 2 \pi \omega a^{2} l B_{0}^{2} \frac{\mu_{r}}{\mu_{0}} \frac{J_{1}(z)\left(z J_{0}(z)-J_{1}(z)\right)^{*}}{\left|z J_{0}(z)+J_{1}(z)\left(\mu_{r}-1\right)\right|^{2}}
\end{aligned}
$$

where $R_{0}=l /\left(\sigma \pi a^{2}\right)$ denotes the DC resistance of the wire. The first, second and third terms in the right hand side of (13) represent change in magnetic energy stored in $\Omega_{\text {out }}$, eddy currents losses $P_{\text {skin }}, P_{\text {prox }}$ due to skin and proximity effects, respectively. The eddy current losses are separated into $P_{\text {skin }}, P_{\text {prox }}$ due to orthogonality as pointed out in [9]. From (13), it is also possible to determine the impedance of the wire. To compute the complex power and impedance, however, we have to know $B_{0}$ which is generated by wire currents surrounding $\Omega_{c}$ and magnetic cores. A numerical method to evaluate $P$ will be given in II.D.

\section{Complex permeability of isolated wire}

By introducing the complex permeability, we can evaluate the eddy currents due to the proximity effect in the wire through magnetostatic field analysis without fine FE discretization. We will derive the complex permeability of the wire on the basis of the above formulation. First of all, the permeability of the wire is assumed to be $\mu_{0}$. Then, the vector potential in $\Omega_{\text {out }}$ generated by the eddy current $J_{z}$ due to the proximity effect, which corresponds to the second term of (4), flowing along the wire shown in Fig.1 satisfies the two-dimensional Poisson equation

$$
\nabla^{2} A_{z}=-\mu_{0} J_{z}
$$

From (14) it follows that

$$
A_{z}(\boldsymbol{r})=-\frac{\mu_{0}}{2 \pi} \int_{\Omega_{c}} J_{z}\left(\boldsymbol{r}^{\prime}\right) \log \left|\boldsymbol{r}-\boldsymbol{r}^{\prime}\right| d S^{\prime}
$$

Now it is assumed that the observation point $\boldsymbol{r}$ is sufficiently far from the source $\boldsymbol{r}^{\prime}$, that is, $|\boldsymbol{r}| \gg\left|\boldsymbol{r}^{\prime}\right|$. Then, we can obtain

$$
A_{z}(\boldsymbol{r})=\frac{\mu_{0}}{2 \pi} \int_{\Omega_{c}} J_{z}\left(\boldsymbol{r}^{\prime}\right) \frac{\boldsymbol{r} \cdot \boldsymbol{r}^{\prime}}{r^{2}} d S^{\prime}
$$

where we use the fact that the integral of $J_{z}$ over $\Omega_{c}$ vanishes. Moreover, the integral of $J_{z} y^{\prime}$ also vanishes because $J_{z}$ is symmetric with respect to the $x$-axis. Thus (16) can be written as

$$
\begin{gathered}
A_{z}(\boldsymbol{r})=-\frac{\mu_{0}}{2 \pi} \frac{m}{r} \cos \theta \\
m=-\int_{\Omega_{c}} J_{z}\left(\boldsymbol{r}^{\prime}\right) r^{\prime} \cos \theta^{\prime} d S^{\prime}
\end{gathered}
$$

where $m$ denotes the magnetic moment parallel to $y$-axis. Equation (16) indicates that only the dipole field remains and the contributions from higher multipole components vanish in far fields when there are no net currents. Simultaneously we conclude that the dipole moment (17b) generates the vector potential written by (17a). If there exists only the eddy current due to the proximity effect, which corresponds to the dipole field represented by the second term of (4), the magnetic field can be written by (17a) even in near fields. Substituting (4) into (17b), where $J_{z}=\sigma E_{z}$, the magnetic moment generated by the eddy current in the wire, which expresses the diamagnetic effect, is obtained as follows:

$$
m=2 \pi \frac{B_{0}}{\mu_{0}} a^{2} \frac{J_{1}(z)-z J_{1}{ }^{\prime}(z)}{J_{1}(z)+z J_{1}{ }^{\prime}(z)}
$$

When the wire is made of magnetic material which has permeability $\mu \neq \mu_{0}$, magnetization current has to be included in $J_{z}$ in (14). Instead of doing so, we evaluate $m$ based on the fact that the first term in (7) expresses the dipole field, which is also represented in terms of $m$ using (17a). This leads to $m=$ $-2 \pi j q_{1} /\left(\mu_{0} \omega\right)$, from which we obtain

$$
m=2 \pi \frac{B_{0}}{\mu_{0}} a^{2} \frac{\mu_{r} J_{1}(z)-z J_{1}{ }^{\prime}(z)}{\mu_{r} J_{1}(z)+z J_{1}{ }^{\prime}(z)}
$$

Obviously, (19) is the extension of (18). The magnetization $M=m /\left(\pi a^{2}\right)$ is readily obtained from (19) as

$$
M=2 \frac{B_{0}}{\mu_{0}} \frac{\mu_{r} J_{1}(z)-z J_{1}{ }^{\prime}(z)}{\mu_{r} J_{1}(z)+z J_{1}{ }^{\prime}(z)}
$$

In the static limit $\omega \rightarrow 0,(20)$ reduces to the magnetization of a cylinder immersed in the magnetostatic field $B_{0}$ [e.g. 16] 


$$
M=2 \frac{B_{0}}{\mu_{0}} \frac{\mu_{r}-1}{\mu_{r}+1}
$$

Here let us define the complex permeability $\dot{\mu}$ of a round wire such that (20) is consistent with (21), that is

$$
\frac{\dot{\mu}_{r}-1}{\dot{\mu}_{r}+1}=\frac{\mu_{r} J_{1}(z)-z J_{1}{ }^{\prime}(z)}{\mu_{r} J_{1}(z)+z J_{1}{ }^{\prime}(z)}
$$

From (22) it follows that

$$
\dot{\mu}_{r}=\mu_{r} \frac{J_{1}(z)}{z J_{1}{ }^{\prime}(z)}
$$

which is the complex permeability of an isolated round wire. The denominator in (23) can also be written as $z J_{0}(z)-J_{1}(z)$. Note that (23) is valid under the assumption that the external magnetic field is nearly uniform.

\section{FE analysis using complex permeability}

The complex number next to $\mu_{r}$ in (23) expresses the diamagnetic property due to the proximity effect. The question now arises: is it possible to correctly compute $P_{\text {prox }}$ using (23)? To answer to this equation, let us evaluate the power in $\Omega_{c}$ using (23). When a round wire, complex permeability $\dot{\mu}$, is immersed in a uniform magnetic field $B_{0}$ as shown in Fig.1, the magnetic field in $\Omega_{c}$ is given by $H=2 B_{0} /\left(\dot{\mu}+\mu_{0}\right)$. Thus the power can be written as

$$
j \omega \int_{\Omega_{c}} \dot{\mu} \frac{|\boldsymbol{H}|^{2}}{2} d v=j \omega \pi a^{2} l \frac{\dot{\mu}}{2}\left|\frac{2}{\dot{\mu}+\mu_{0}}\right|^{2} B_{0}^{2}
$$

By substituting (23) into the right hand side of (24), we find that (24) equals the third term of (13). Hence, the complex permeability gives the complex power which is consistent with that obtained from the energy conservation law. This is the consequence of the fact that the dipole field generated by the eddy currents due to the proximity effect is correctly represented by (23). From this proposition, it is concluded that the whole power can be computed from

$$
P=\frac{j \omega}{2} \int_{\Omega_{c}+\Omega_{\text {out }}} \mu|\boldsymbol{H}|^{2} d v+Z_{\text {skin }}|I|^{2}
$$

where $\mu$ is set to $\dot{\mu}$ in the wire, and $Z_{\text {skin }}=R_{0} z J_{0}(z) / 4 J_{1}(z)$ which approaches $R_{0} / 2$ in the static limit. The real and complex parts of the first term in (25) represent the sum of $P_{\text {prox }}$ and time variation in the stored magnetic energy, while the second term represents $P_{\text {skin }}$ including the DC Joule loss. It is clear in light of the above discussions that (25) is valid even when there are multiple wires.

The first term in (25) can be evaluated by performing FE analysis of magnetostatic field assuming that the external magnetic field is nearly uniform in the scale of the wire diameter. The FE equation for two-dimensional fields can be written in the form

$$
\mathrm{K} \boldsymbol{a}=\boldsymbol{b}
$$

where the components in $\mathrm{K}$ and $\boldsymbol{b}$ are given by

$$
K_{i j}=\int_{\Omega_{c}+\Omega_{\text {out }}} \frac{1}{\mu} \nabla w_{i} \cdot \nabla w_{j} d S, \quad b_{i}=\frac{I}{S_{c}} \int_{\Omega_{c}} w_{i} d S
$$

In (26) and (27), $\boldsymbol{a}$ denotes the vector containing the nodal values of $A_{z}$. The permeability $\mu$ in (27) is again set to $\dot{\mu}$ in the wire. When there are other conductors, the eddy current term is added to $K_{i j}$. The current in the right hand side of (26) represents the imposing current which is assumed uniform. The diamagnetic effect due to the eddy currents in the wire is represented by the complex permeability. Note here that the wire cross sections can be subdivided into FEs without considering the skin depth in contrast to the conventional FE analysis. Hence the number of unknowns in (26) can be reduced.

When we consider the voltage input problem, the current is determined by solving the FE equation coupled with the circuit equation as

$$
\left[\begin{array}{cc}
\mathrm{K} & -\boldsymbol{b} \\
-\boldsymbol{b}^{t} & j Z_{e} / \omega
\end{array}\right]\left[\begin{array}{c}
\boldsymbol{a} \\
I
\end{array}\right]=\left[\begin{array}{c}
0 \\
j V / \omega
\end{array}\right]
$$

The three-dimensional FE equation can also be derived in a similar way. When the number of turns is large, the FE discretization of each wire cross-sections would still result in a large equation system. To circumvent this problem, homogenization introduced below is effective.

\section{E. Homogenization}

The homogenized permeability of magnetic composite can be evaluate using the Ollendorff formula [12]

$$
\left\langle\mu_{r}\right\rangle=1+\frac{\eta\left(\mu_{r}-1\right)}{1+N(1-\eta)\left(\mu_{r}-1\right)}
$$

where the diamagnetic constant $N$ is $1 / 2$ for round wires. Although (29) has been introduced in [12], a brief derivation is given in Appendix A for completeness of this paper. Moreover it can be shown that (29) is essentially equivalent to the Clausius-Mossotti formula, which is shown in Appendix B.

For magnetostatic fields, the homogenized permeability computed from (29) has been shown to agree well with that computed from the FE analysis of an elementary cell under the periodic boundary condition $[17,18]$. We here extend (29) to evaluate the homogenized complex permeability $\left\langle\dot{\mu}_{r}>\right.$ of the multi-turn coil in time-harmonic fields. That is, using (23), (29), the homogenized complex permeability of the multi-turn round coil is computed from

$$
<\dot{\mu}_{r}>=1+\frac{2 \eta\left(\mu_{r} \frac{J_{1}(z)}{z J_{1}^{\prime}(z)}-1\right)}{2+(1-\eta)\left(\mu_{r} \frac{J_{1}(z)}{z J_{1}^{\prime}(z)}-1\right)}
$$


Validity of this extension is clear in light of the derivation of (29) given in Appendix A. The profile of $\left\langle\dot{\mu}_{r}\right\rangle$ is plotted as a function of the wire radius normalized with the skin depth, $a / \delta$, in Fig.2, where $\mu_{r}=1$ is assumed. The curves for $\eta=1$ are plotted as a limiting case which cannot be realized when using round wires.

Now it is possible to treat the multi-turn coil as a homogenous material whose permeability is $\left\langle\dot{\mu}_{r}\right\rangle$. The computational time to solve (26) or (28) can be significantly reduced especially when the number of turns is large. The impedances computed by the proposed method will be compared with those obtained by numerical computations and experiments in the next section.

\section{NUMERICAL AND EXPERIMENTAL RESULTS}

The multi-turn copper coil, wound around a core of permeability $\mu_{\text {core }}$, placed above a copper disk, shown in Fig. 3 is analyzed using the proposed method and conventional FEM. The copper conductivity is set to $5.7610^{7} \mathrm{~S} / \mathrm{m}$. In the latter analysis, the quasi-static Maxwell equations (1) are solved by discretizing each wire into fine elements so that the element size
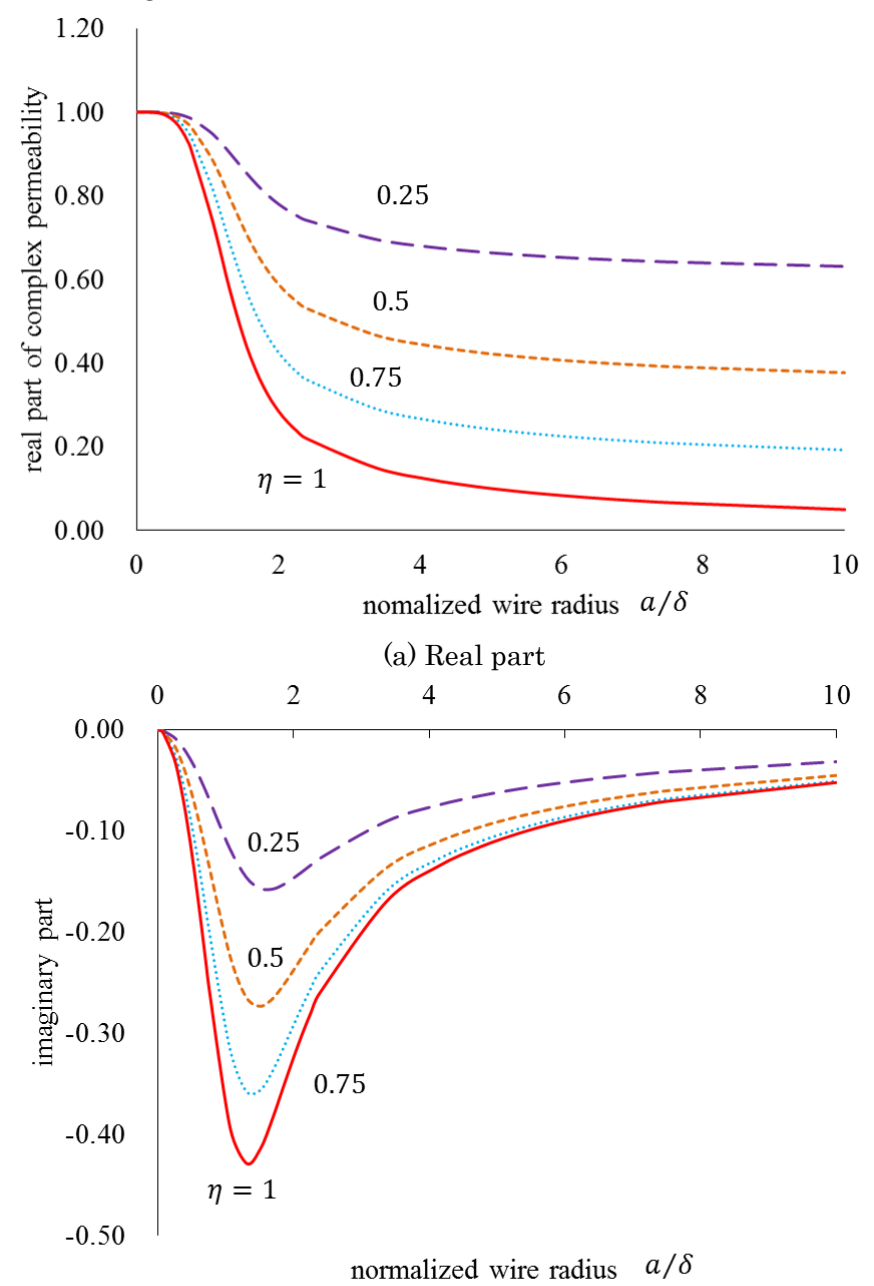

(b) Imaginary part

Fig.2 Dependence of homogenized permeability $<\dot{\mu}_{r}>$ on normalized radius $a / \delta$, where denotes $\delta$ skin depth. is sufficiently smaller than the skin depth. In the proposed analysis, the magnetostatic problem (26) is solved where the coil region in Fig.3 is modeled as a homogenous material of $<$ $\dot{\mu}_{r}>$. In both analyses, the imposing current is assumed to be given. The impedance of the coil, $Z=2 P / I^{2}$, is computed from (25) in the proposed analysis, while in the conventional FE analysis it is obtained from the complex power $P$ given by (11). When computing $P$ from (25), the permeability of the coil region is set to $\left\langle\dot{\mu}_{r}\right\rangle$. The number of elements in the coil region are 35240 and 740 for the conventional and proposed $\mathrm{FE}$ analyses.

Fig.4 shows the dependence of the impedance $Z$ of the multiturn copper coil on the normalized wire radius when $\mu_{\text {core }}=\mu_{0}$ and $\mu_{\text {core }}=1000 \mu_{0}$. The proposed method provides almost the same results as those obtained by the conventional FE analysis. The difference between both results is found to be lower than $2 \%$. This good correspondence would be due to the fact that the local field, which is $\boldsymbol{H}_{l o c}$ in (A1), acting on a wire is rather uniform. We expect this uniformity considering the fact that the magnetic field in a cylindrical hole in a cylindrical conductor along which a constant current flows is uniform [15, sec.30].

The AC resistance $\operatorname{Re}(\mathrm{Z})$ of a 50-turn enameled copper coil, conductor radius $0.15 \mathrm{~mm}$, cover thickness $0.03 \mathrm{~mm}$, shown in Fig. 5, is computed using the proposed method. The coil is wound around a bobbin made of photocurable resin, PRH35ST2, whose radius is $30 \mathrm{~mm}$. The volume fraction is evaluated

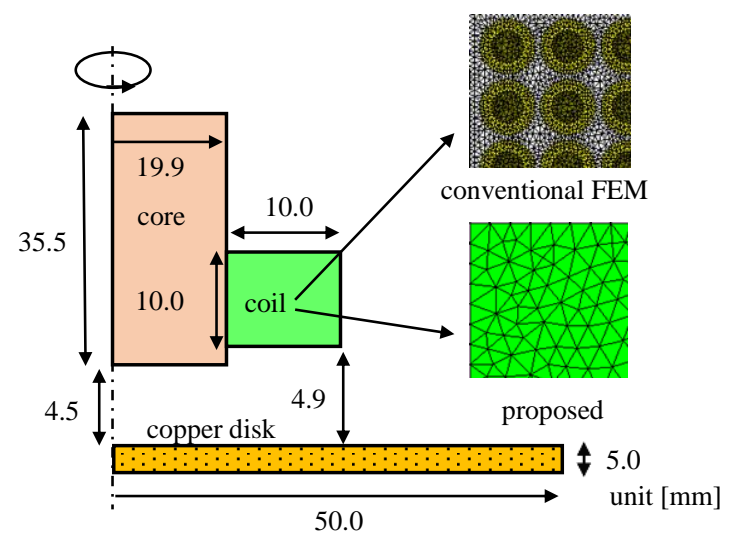

Fig. 3 Multi-turn copper coil placed above a conducting disk. The system is assumed axisymmetric. The coil radius and air gap between coils are 0.5 and $0.1 \mathrm{~mm}$, and number of turn is 81 so that $\eta=0.64$. The FE meshes in a part of the coil region for the conventional and proposed FE analyses are shown .

to be $62.9 \%$. The AC resistance is measured using an LCR meter, HIOKI IM3523. The results are plotted in Fig.6. The relative error of the computational result is less than $3 \%$. The computed and measured inductances are about $0.32 \mathrm{mH}$. If the number of turn is further increased, the effect of electrostatic capacitance among the coil turns becomes non-negligible. Numerical evaluation of this effect remains as our future work.

\section{CONCLUSIONS}

In this paper, a semi-analytical approach to analyze the electromagnetic property of multi-turn coils has been presented. The homogenized complex permeability of the multi-turn coil, 
which depends on the wire conductivity, permeability and volume fraction, has been analytically derived. The impedance and loss of the multi-turn coil can be computed from magnetostatic field analysis where the coil region is modeled as a uniform material with the homogenized complex permeability. By natural extension of the proposed method, electromagnetic properties of the magnetic plated wires could also be analyzed. Numerical evaluation of electrostatic capacitance among multiturn coil as well as analysis of twisted and woven Litz wires remain as our future work.

\section{APPENDIX A}

Here the Ollendorff formula (29) is derived. Let us consider a composite composed of magnetic particles with permeability $\mu$, diamagnetic constant $N$ and volume fraction $\eta$ which are embedded in non-magnetic medium. The macroscopic magnetic field, magnetic induction and magnetization are denoted by $\boldsymbol{H}, \boldsymbol{B}$ and $\boldsymbol{M}$.

The local field $\boldsymbol{H}_{l o c}$ in which the magnetic particle is immersed can be obtained by taking the particle away. That is,

$$
\boldsymbol{H}_{l o c}=\boldsymbol{H}+N \boldsymbol{M}
$$

The magnetic field $\boldsymbol{H}_{\text {in }}$ inside the particle can be expressed as

$$
\boldsymbol{H}_{\text {in }}=\boldsymbol{H}_{\text {loc }}-N \boldsymbol{M}_{\text {in }}
$$

where $\boldsymbol{M}_{i n}$ is the magnetization inside the particle which is, by definition, given by

$$
\boldsymbol{M}_{\text {in }}=\left(\mu_{r}-1\right) \boldsymbol{H}_{\text {in }}
$$

It follows from (A1)-(A3) that

$$
\boldsymbol{M}_{\text {in }}=\frac{\mu_{r}-1}{1+N\left(\mu_{r}-1\right)} \boldsymbol{H}_{l o c}
$$

By averaging the magnetization over the particle and nonmagnetic medium, the macroscopic magnetization $\boldsymbol{M}$ is obtained as

$$
\boldsymbol{M}=\frac{\eta\left(\mu_{r}-1\right)}{1+N\left(\mu_{r}-1\right)} \boldsymbol{H}_{l o c}
$$

Substituting (A5) into (A1), we find

$$
\boldsymbol{M}=\frac{\eta\left(\mu_{r}-1\right)}{1+N(1-\eta)\left(\mu_{r}-1\right)} \boldsymbol{H}
$$

The coefficient in (A6) is the homogenized magnetic susceptibility $\langle\chi\rangle$. The homogenized permeability is given by $\left\langle\mu_{r}\right\rangle=1+\langle\chi\rangle$ which is the Ollendorff formula (29). In the proposed method, the complex permeability $\dot{\mu}_{r}$ is defined so that (A4), which corresponds to (20) and (21) in the static limit, holds. Hence use of $\dot{\mu}_{r}$ in stead of $\mu$ in (29) is valid.

\section{APPENDIX B}

The Clausius-Mossotti formula is widely used to evaluate the macroscopic permittivity of nonpolar molecules [e.g. 19] . Here the magnetic counterpart of Clausius-Mossotti formula will be shown essentially equivalent to the Ollendorff formula (29).

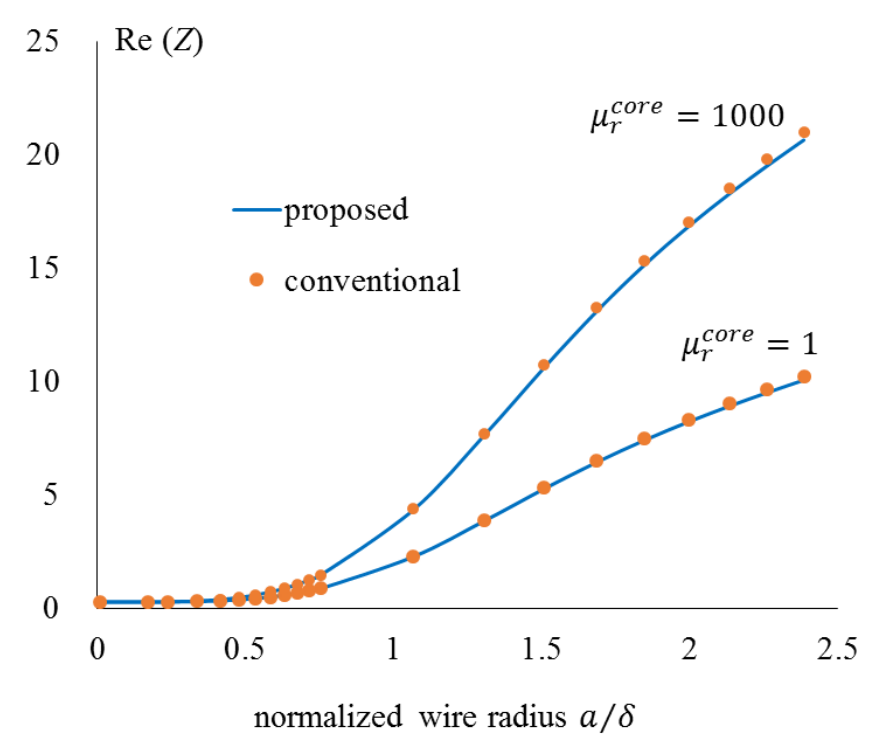

(a) Real part

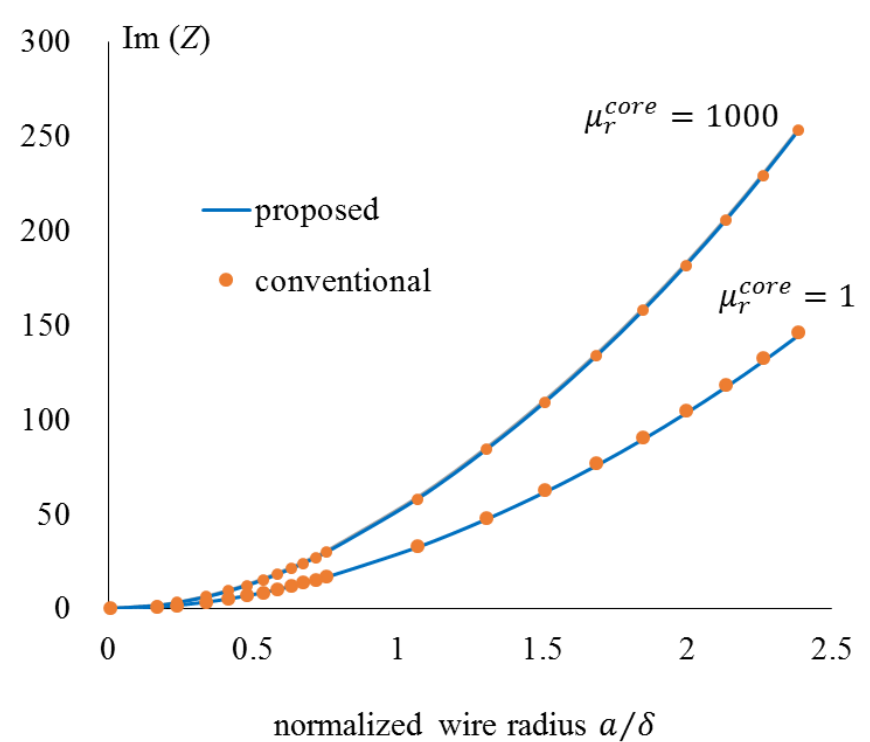

(b) Imaginary part

Fig. 4 Impedance $Z[\Omega]$ of the copper multi-turn coil shown in Fig.3 plotted against normalized wire radius. 


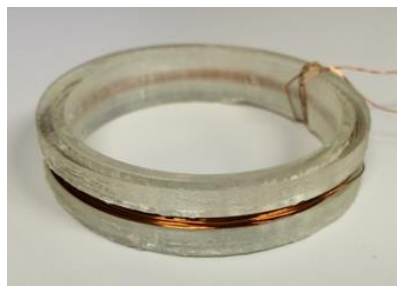

Fig. 5 50-turn enameled copper coil, with conductor radius 0.15 $\mathrm{mm}$, cover thickness $0.03 \mathrm{~mm}$. The coil is wound around a bobbin, made by a photocurable $3 \mathrm{D}$ printer, whose radius is $30 \mathrm{~mm}$.

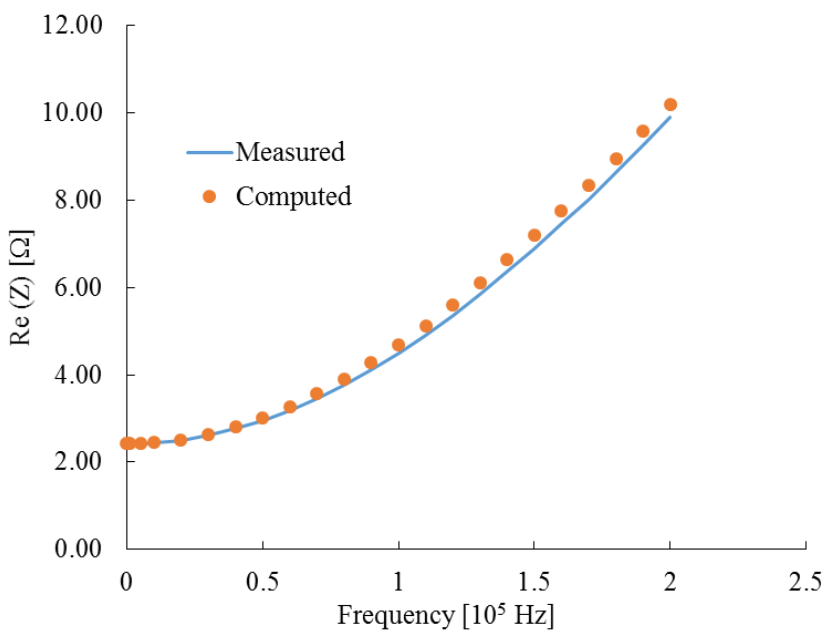

Fig. 6 AC resistance of 50-turn coil shown in Fig.5.

The molecules in the Clausius-Mossotti theory are replaced by the magnetic particles with permeability $\mu$, diamagnetic constant $N$ embedded in non-magnetic medium. The magnetic moment of the particle, number density and polarizability are denoted by $\boldsymbol{m}, n$ and $\alpha$, respectively. Because the local magnetic field, called Lorentz field, is given by (A1), the macroscopic magnetization, $\boldsymbol{M}=n \boldsymbol{m}$, is given by

$$
\boldsymbol{M}=n \alpha \boldsymbol{H}_{l o c}=n \alpha(\boldsymbol{H}+N \boldsymbol{M})
$$

Inserting (B1) into $\boldsymbol{B}=\mu_{0}(\boldsymbol{H}+\boldsymbol{M})$, we have the macroscopic relative permeability

$$
<\mu_{r}>_{\mathrm{CM}}=1+\frac{n \alpha}{1-N n \alpha}
$$

The magnetic moment in a magnetic particle immersed in the local field is given by

$$
\boldsymbol{m}=\Delta V \frac{\left(\mu_{r}-1\right)}{1+N\left(\mu_{r}-1\right)} \boldsymbol{H}_{l o c}
$$

where $\Delta V$ denotes the volume of the particle. The number density can be expressed in terms of the volume fraction $\eta$ as $n=\eta / \Delta V$. Since the coefficient in (B3) corresponds to $\alpha$, we obtain

$$
n \alpha=\eta \frac{\mu_{r}-1}{1+N\left(\mu_{r}-1\right)}
$$

Substituting (B4) into (B2), we find that $\left\langle\mu_{r}\right\rangle_{\mathrm{CM}}=\left\langle\mu_{r}>\right.$.

Similarly, the Maxell-Garnett formula, widely used in microwave engineering [e.g. 20], for spherical particles $(N=$ $1 / 3)$

$$
\frac{\langle\mu\rangle_{\mathrm{MG}}-\mu_{0}}{\langle\mu\rangle_{\mathrm{MG}}+2 \mu_{0}}=\eta \frac{\mu-\mu_{0}}{\mu+2 \mu_{0}}
$$

can also be shown equivalent to (29).

\section{ACKNOWLEDGMENT}

The author thanks Dr. K. Kawano of Taiyo Yuden Co. for his helpful comments, and Mr. S. Fujita, Y. Otomo and Mr. Y. Ito of Hokkaido University for their support for numerical computations and experimental measurements. This work was supported in part by KAKENHI Grant Number 15H02976.

\section{REFERENCES}

[1] P. H. Mellor, R. Wrobel and N. McNeill, "Investigation of Proximity Losses in a High Speed Brushless Permanent Magnet Motor," 41st IAS Annual Meeting, Industry Applications Conference, 2006.

[2] M. Popescu and D. G. Dorrell, "Proximity Losses in the Windings of High Speed Brushless Permanent Magnet AC Motors With Single Tooth Windings and Parallel Paths," IEEE Trans. Industrial Electronics., vol.49, no.7, 2013, pp.3913-3916.

[3] R. Wrobel, A. Mlot and P. H. Mellor, "Contribution of End-Winding Proximity Losses to Temperature Variation in Electromagnetic Devices," IEEE Trans. Magn., vol.59, no.2, 2011, pp.848-857.

[4] Z. Yang, W. Liu and E. Basham, "Inductor Modeling in Wireless Links for Implantable Electronics," IEEE Trans. Magn., vol.43, no.10, 2007, pp.3851-3860.

[5] J. Acero1, P. J. Hernández, J. M. Burdío1, R. Alonso, and L. A. Barragán, "Simple Resistance Calculation in Litz-Wire Planar Windings for Induction Cooking Appliances," IEEE Trans. Magn., vol.41, no.4, 2005, pp.1280-1288.

[6] C. V. Dodd and W. E. Deeds "Analytical Solutions to Eddy - Current Probe - Coil Problems," J. App. Phys., vol.39, no.6, 1968, pp.2829-2838.

[7] P.L. Dowell, "Effects of eddy currents in transformer windings", Proceedings of the IEEE, vol. 113, no. 8, 1966, pp. 1387-1394.

[8] Xi Nan and C. R. Sullivan, "An Improved Calculation of Proximity-Effect Loss in High-Frequency Windings of Round Conductors," IEEE 34th Annual Power Electronics Specialist Conference, vol.2, 2003, pp.853-860

[9] J. A. Ferreira, "Improved analytical modeling of conductive losses in magnetic components", IEEE Trans. Power Electronics, vol. 9, no.1, 1994, pp. 127-131.

[10] A. Podoltsev and B. Lebedev, "Analysis of effective resistance and eddy current losses in multiturn winding of high-frequency magnetic components," IEEE Trans. Magn., vol. 39, no. 1, 2003, pp. 539-548.

[11] J. Gyselinck and P. Dular, "Frequency-domain homogenization of bundles of wires in 2-D magnetodynamic FE calculations," IEEE Trans. Magn., vol. 41, no. 5, 2005, pp. 1416-1419.

[12] F. Ollendorff, "Magnetostatik der Massekerne," Arch.f. Elektrotechnik., 25, 1931, pp. 436-447.

[13] A. Buffa, H. Ammari, and J. C. Nédélec, A Justification of Eddy Currents Model for the Maxwell Equations, SIAM J. Appl. Math., vol.60, no.5, 2000, pp.1805-1823.

[14] K. Schmidt, O. Sterz and R. Hiptmair, Estimating the eddy-current modelling error, IEEE Trans. Magn., vol. 44, no.6, 2008, 686-689.

[15] L D Landau, L. P. Pitaevskii and E.M. Lifshitz, Electrodynamics of Continuous Media, 2nd ed., Butterworth-Heinemann, 1984.

[16] J. A. Stratton, Electromagnetic Theory, section 4.20, 1941.

[17] H. Waki and H. Igarashi, "Estimation of effective permeability of magnetic composite materials", IEEE Trans. Magn., 41, no. 5, 2005, pp. $1520-1523$. 
[18] Y. Ito and H. Igarashi, "Computation of macroscopic electromagnetic properties of soft magnetic composite", IEEE Trans. Magn., 49, no.5, 2013, pp. 1953-1956.

[19] R. P. Feynman, R. B. Leighton and M. Sands, The Feynman Lectures on Physics, vol. II, 1970

[20] A. Berthault, D. Rousselle and G. Zerah, "Magnetic properties of Permalloy microparticles", J. Magn Magn. Mater., vol.112, 1992, pp.477480 .

H. Igarashi (M'94-) received the B.E. and M.E. degrees in electrical engineering from Hokkaido University, Sapporo, Japan, in 1982 and 1984, respectively, and the $\mathrm{Ph} . \mathrm{D}$. degree in engineering from Hokkaido University in 1992. He has been a professor at the Graduate School of Information Science and Technology, Hokkaido University, since 2004. He has worked as a research engineer at Canon Co. Ltd., from 1984 to 1989. From 1989 to 1999, he was a research associate with the Faculty of Engineering, Hokkaido University. He was a guest researcher at Berlin Technical University, Germany, under support from the Humboldt Foundation from 1995-1997. He was an associate professor from 1999 to 2004 at Kagawa University, Japan, and Hokkaido University. His research area is computational electromagnetism, design optimization and RFID technologies. He has authored and coauthored more than 100 peerreviewed journal papers. 